\title{
Equipamiento y uso de Tecnologías de la Información y Comunicación (TIC) en los hogares españoles durante el periodo de confinamiento. Asociación con los hábitos sociales, estilo de vida y actividad física de los niños menores de 12 años
}

\begin{abstract}
Equipment and use of Information and Communication Technologies (ICT) in Spanish homes during the period of confinement. Association with the social habits, lifestyle and physical activity of children under 12 years old
\end{abstract}

Víctor Arufe Giráldez. Universidad de A Coruña. España.

v.arufe@udc.es

[CV] $\mathrm{C}$ R $\mathrm{R}$

Javier Cachón Zagalaz. Universidad de Jaén. España.

jcachon@ujaen.es

$[\mathrm{CV}]$ (1)

María Luisa Zagalaz Sánchez. Universidad de Jaén. España

lzagalaz@ujaen.es

[CV] $61 \%$

Alberto Sanmiguel-Rodríguez. Universidad Antonio de Nebrija. Universidad Camilo José Cela.

España

asrgz2014@gmail.com

[CV] $\bigcirc$

Gabriel González Valero. Universidad de Jaén. España.

ggvalero@ujaen.es

$[\mathrm{CV}]$ (1)

Cómo citar este artículo / Referencia normalizada

Arufe Giráldez, V., Cachón Zagalaz, J., Zagalaz Sánchez, Ma. L., Sanmiguel-Rodríguez, A. y González-Valero, G. (2020). Equipamiento y uso de Tecnologías de la Información y Comunicación (TIC) en los hogares españoles durante el periodo de confinamiento. Asociación con los hábitos sociales, estilo de vida y actividad física de los niños menores de 12 años. Revista Latina de Comunicación Social, 78, 183-204. https://www.doi.org/10.4185/RLCS-2020-1474 


\title{
RESUMEN
}

Introducción: Las TIC están presentes en muchos hogares estableciendo cambios en la comunicación social y estilo de vida. En el año 2020 se ha declarado en España el estado de alarma con un importante periodo de confinamiento debido a la aparición de una nueva pandemia mundial COVID-19. A través de este trabajo se realiza una radiografía del equipamiento y uso de las TIC en los hogares españoles con niños menores de 12 años durante el periodo de confinamiento y cómo ese tiempo y las TIC han influido en los hábitos sociales de los niños, su estilo de vida y nivel de actividad física. Metodología: Se realizó una investigación cuantitativa con un diseño no experimental, de carácter descriptivo, comparativo y correlacional, con una única medición en un solo grupo. La muestra estuvo compuesta por 837 niños españoles menores de 12 años. Resultados: Se confirma un número importante de dispositivos electrónicos en los hogares, tiempos de uso diferentes para cada uno y la existencia de diversas correlaciones entre TIC y hábitos sociales y de estilo de vida de los niños. Discusión: Otros trabajos confirman valores similares de dispositivos electrónicos en los hogares, así como el sobreuso de algunos de ellos en detrimento de la actividad física. Conclusiones: El periodo de confinamiento ha provocado cambios notables en el estilo de vida de los niños y sus hábitos sociales. La presencia de un mayor o menor número de TIC en el hogar y su uso puede influir en otras actividades realizadas por los niños.

PALABRAS CLAVE: TIC; estilo de vida; niños; actividad física; COVID-19; confinamiento: hábitos sociales.

\begin{abstract}
Introduction: ICTs are present in many homes establishing changes in social communication and lifestyle. In 2020, the state of alarm was declared in Spain with a significant period of confinement due to the appearance of a new global pandemic COVID-19. Through this work, an X-ray of the equipment and use of ICT in Spanish homes with children under 12 years of age during the period of confinement and how this period and ICT have influenced children's social devices, their style, are carried out. of life and level of physical activity. Methodology: A quantitative investigation was carried out with a non-experimental design, descriptive, comparative, and correlational character, with a single measurement in a single group. The sample was made up of 837 Spanish children under 12 years of age. Results: The results confirm an important number of electronic devices in homes, different times of use for each device and the existence of various correlations between ICT and children's social and lifestyle habits. Discussion: Other works confirm similar values of electronic devices in homes as well as the overuse of some of them to the detriment of physical activity. Conclusions: The period of confinement has caused notable changes in the children's lifestyle and their social habits. The presence of a mayor or fewer ICTs in the home and their use can influence other activities carried out by children.
\end{abstract}

KEYWORDS: ICT; Lifestyle; children; physical activity; COVID-19; confinement: social habits.

\section{CONTENIDO}

1. Introducción. 1.1. Las Tecnologías de la Información y Comunicación (TIC). 1.2. Hábitos sociales, estilo de vida y de actividad física de los niños menores de 12 años. 1.3. El periodo de confinamiento en España en el año 2020. 2. Objetivos. 3. Metodología. 3.1. Diseño del estudio y participantes. 3.2. Instrumento de recogida de datos y variables. 3.3. Procedimiento. 3.4. Aspectos éticos. 3.5. Análisis de datos. 4. Resultados. 5. Discusión. 6. Conclusiones. 7. Recomendaciones y perspectivas de futuro. 8. Bibliografía. 9. Currículum Vitae. 


\section{Introducción}

\subsection{Las Tecnologías de la Información y Comunicación (TIC)}

Las Tecnologías de la Información y Comunicación (TIC) han evolucionado notablemente en los últimos años. El término TIC puede hacer referencia a cualquier dispositivo electrónico que pueda generar una canal de comunicación o almacenar, gestionar o aportar datos vinculados con la información. Algunos autores como Marqués, P. (2012) otorgan hasta un total de 9 funciones a las TIC, constituyendo estas: un medio de expresión, un canal de comunicación, un instrumento para procesar la información, una fuente abierta de información, un instrumento para la gestión, una herramienta de diagnóstico, un medio didáctico, un generador de nuevos escenarios formativos y un medio lúdico para el desarrollo cognitivo

Se pueden incluir en este concepto los televisores, los teléfonos móviles/smartphones, las tablets, los ordenadores o las videoconsoloas, entre otros dispositivos. Las TIC están presentes en la vida de millones de personas y en múltiples contextos, desde educativos, a familiares, empresariales o sanitarios. Su gran uso, y en ocasiones abuso, han animado a diversos autores a establecer instrumentos de investigación que puedan detectar adicciones, o problemas de conducta, como consecuencia del sobreuso de algunas TIC, a fin de establecer planes de prevención y una actuación rápida (Chamarro Lusar et al., 2014; Cuesta Cambra, Cuesta Díaz, Martínez, \& Niño González, 2020; Hernández Pérez, 2019; Justicia Justicia \& Villadangos González, 2010; Lloret Irles \& Morell Gomis, 2016; Lloret Irles, Morell Gomis, Marzo Campos, \& Tirado González, 2018; Lozano Sánchez et al., 2019; Tejeiro, 2001). Inclusive, organizaciones internacionales como la Organización Mundial de la Salud (OMS) han publicado por primera vez en su historia un informe alertando del abuso de pantallas en la población infantil de 0 a 5 años y de la inactividad física en estas edades, recomendando a la población hacer un uso medido de los dispositivos tecnológicos y evitándolos especialmente en niños menores de 2 años (Guthold, Stevens, Riley, \& Bull, 2018). Ortiz, M.A. et al. (2019) destacan el importante papel de la familia como mediadora del consumo de dispositivos electrónicos de pantalla en la infancia y niñez.

Los dispositivos tecnológicos han irrumpido en la sociedad actual invadiendo los hogares de muchos niños a través de televisores, tablets, ordenadores, videoconsolas y teléfonos móviles. Según datos del Instituto Nacional de Estadística, en el año 2019, 9 de cada 10 hogares españoles contaban con acceso a internet, 8 de cada 10 con ordenador, 5,6 de cada 10 con tablet, 9,8 de cada 10 con teléfono móvil y 9,9 de cada 10 con televisión. (Instituto Nacional de Estadística, 2019). Estamos ante una era digital que conduce a diferentes cambios en los patrones de comunicación personal y social (Lucas Marín, 2006), así como en sectores tan destacados como la política (Solito \& Sorrentino, 2018) o la educación (Gil-Quintana \& Cano Alfaro, 2020; Sánchez López, Pérez Rodríguez, \& Fandos Igado, 2019).

\subsection{Hábitos sociales, estilo de vida y de actividad física de los niños menores de 12 años}

El día a día de la gran mayoría de los niños presenta un patrón común en lo que se refiere a sus hábitos sociales, estilo de vida y de actividad física. Prácticamente la totalidad de los menores de 12 años en España acuden a la escuela todas las mañanas, permaneciendo en ella hasta primera hora de la tarde o, en algunos casos, media tarde. A los 16 años, edad teórica de educación postobligatoria, disminuye la tasa de escolarización situándose alrededor de un $92 \%$ según datos del último informe educativo (Ministerio de Educación y Formación Profesional, 2019). Las tardes de los niños suelen ser ocupadas con múltiples actividades, que inciden en su desarrollo social, afectivo, psíquico y físico. De forma general, con porcentajes de población escolar de entre el 58 y el 87\%, diversos estudios confirman que los menores de 12 años realizan una cantidad mínima de actividad física con 
amigos en escuelas deportivas (Barja Fernández, Pino Juste, Portela Pino, \& Leis Trabazo, 2020; Torres Moreno, Solera Albero, Sahuquillo Martínez, \& Tárraga López, 2020; Zueck, Ramírez García, Rodríguez Villalobos, \& Irigoyen Gutiérrez, 2020). Sin embargo, estos niveles de práctica de actividad física distan en muchos casos de la cantidad de actividad física recomendada por diversas instituciones para la adquisición de un estilo de vida saludable (Piercy et al., 2018; OMS 2019) Concretamente la OMS (2010), indica que los niños y jóvenes de 5 a 17 años deben invertir, como mínimo, 60 minutos diarios en actividades físicas de intensidad moderada a vigorosa. Más de 60 minutos diarios de actividad física tendrán un beneficio aún mayor para la salud y debería ser, en su mayor parte, aeróbica. Asimismo, convendría incorporar, como mínimo tres veces por semana, actividades vigorosas que refuercen, en particular, los músculos y huesos. Otra de las actividades sociales que desarrollan los niños en su tiempo libre es el juego libre realizado en la calle o en el parque, confirmando algún reciente estudio porcentajes de participación en menores de 12 años del 23\% de los niños (Jover Olmeda, Camas Garrido, Martín-Ordanza Santos, \& Sánchez Serrano, 2018).

Las tareas escolares, independientemente de que sean un deber o un placer para los niños, constituyen otra rutina adquirida por prácticamente la totalidad de los estudiantes con la existencia de diversos trabajos que han abordado este aspecto (Valle et al., 2019). Incluso recientes estudios internacionales confirman un mayor compromiso con los deberes y tiempos dedicados a las tareas en niños cuyos progenitores tienen un mayor nivel socioeconómico (Nakamura, Yamashita, Akabayashi, Tamura, \& Zhou, 2020). España es el quinto país de la Organización para la Cooperación y Desarrollo Económicos (OCDE) en el que los niños invierten una mayor cantidad de horas a las tareas escolares en casa, 6,5 a la semana frente a las 4,8 de media del resto de países (Salinas, 2014). Pero estos también tienen tiempo para hacer uso y disfrute de diversos dispositivos tecnológicos en sus hogares (Boente Antela, Leirós Rodríguez, \& García Soidán, 2020).

Además de estos hábitos sociales existen otros también estudiados en la literatura y que adquieren los menores en menor medida, tales como la participación en las tareas domésticas, con influencia de la variable de género en su distribución (García, 2017; Paterna Bleda \& Martínez Martínez, María del Carmen, 2009; Rodríguez Hernández \& Santana Bonilla, 2006; Scarafoni, 2016), confirmando algunos trabajos que, cuando los niños participan en la economía doméstica, desarrollan ciertas habilidades que contribuyen al mantenimiento de un estilo de vida centrado en la gestión de recursos locales (Remorini, Teves, Palermo, Jacob, \& Desperés, 2019). En otra investigación realizada con una muestra de niños de entre 6 y 10 años se confirmó que su rendimiento para completar las tareas en el hogar mejora en el transcurso de la infancia media y este desempeño se predice por su atención a las instrucciones y su función ejecutiva (Perone, Anderson, \& Youatt, 2020).

Otra de las rutinas instauradas en el estilo de vida de muchos niños son los hábitos de lectura con porcentajes de niños lectores frecuentes del $72 \%$. Si bien es cierto que en ocasiones las lecturas realizadas por estos son asignadas como obligatorias desde la escuela (Yubero Jiménez \& Larrañaga Rubio, 2010). Alguna investigación señala que la afición y conducta lectora tienen valores óptimos en niños de 9-13 años descendiendo entre los 14 y 16 años y recuperándose en Bachillerato (Fiz Poveda, Olea Aisa, Goicoechea Tabar, \& Ibiricu Díaz, 2000).

En algunos casos invierten tiempo en aprender a tocar un instrumento, hallándose en estudios recientes resultados muy positivos entre el aprendizaje de la música en la infancia y la mejora de las funciones cognitivas y rendimiento académico, especialmente cuando se trata de música instrumental (Capistrán Gracia, 2020; Guhn, Emerson, \& Gouzouasis, 2020). 
Una de las actividades con un marcado carácter social es el juego en familia, aunque en la actualidad están presentes diferentes factores que reducen el tiempo de juego en familia, tales como: un estilo de vida apresurado, cambios en la estructura familiar y mayor atención a actividades académicas (Ginsburg, and Committee on Communications, \& and Committee on Psychosocial Aspects of Child and Family Health, 2007) o la preferencia por invertir el tiempo libre en el uso de dispositivos tecnológicos (Fumero, Marrero, Bethencourt, \& Peñate, 2020). El juego en familia se presenta como un hábito muy enriquecedor para los niños y progenitores. Algunos estudios afirman una clara asociación entre los niveles de felicidad de las familias y el tiempo que pasan jugando con los niños. Así, 9 de cada 10 familias que pasan más de cinco horas jugando juntos cada semana se describen a sí mismos como felices. (Christiansen, 2018). También el juego libre es importante para potenciar diferentes habilidades como el proceso de pensamiento o la creatividad, especialmente si en este juego libre está presente cierta cantidad óptima de actividad física (Piya-Amornphan, Santiworakul, Cetthakrikul, \& Srirug, 2020). Las funciones cognitivas mejoran igualmente con el juego libre (Barker et al., 2014) señalando este último estudio que los niños cuyos progenitores les planifican y estructuran todas sus actividades diarias sin ofrecerles tiempo para este tipo de juego tienen un menor desarrollo de sus capacidades cognitivas, menor iniciativa y resolución de problemas. Otro trabajo también confirma que la oportunidad y la promoción del juego libre en la infancia predicen significativamente algunos indicadores de éxito social en la vida adulta (Greve, Thomsen, \& Dehio, 2014).

Por todo ello se puede constatar que el estilo de vida de los niños, aunque puede ser diferente, tiene patrones muy similares en relación con las actividades y tareas posibles que puede incorporar en las rutinas de su día a día. Las diferencias se establecerán en función de la elección de las tareas y de la inversión de tiempo que haga en cada una de ellas. Pero este estilo de vida puede verse alterado si a los niños se les priva de la libertad, de poder salir de su casa, y por ello se considera importante abordar este contexto novedoso como es el periodo de confinamiento establecido por el Gobierno de España y que supuso una alteración del estilo de vida de muchos niños.

\subsection{El periodo de confinamiento en España en el año 2020}

España y una gran cantidad de países han experimentado una situación excepcional a principios del año 2020. Previamente, en concreto el día 5 de enero del 2020 la Organización Mundial de la Salud (OMS) hacía público su primer parte sobre brotes epidémicos relativo al nuevo virus, una publicación técnica de referencia para la comunidad mundial de investigación y salud pública y los medios de comunicación (Organización Mundial de la Salud, 2020). Dos meses más tarde, el 14 de marzo el Gobierno de España decretaba el estado de alarma en todo el territorio español debido a la pandemia registrada por la aparición de un nuevo virus, coronavirus COVID-19 (Gobierno de España, 2020). Este hecho provoca un parón en las actividades diarias de todos los españoles, en su comunicación y en cambios importantes de su estilo de vida y actividad laboral.

Con la declaración de estado de alarma se establece un periodo de confinamiento "cuarentena" que paraliza la vida social de todas las familias españolas, teniendo que estar recluidas en sus hogares sin poder salir a la calle, salvo casos excepcionales. Es sin lugar a duda un periodo que prácticamente la gran mayoría de la población no había vivido o experimentado anteriormente.

Durante la "cuarentena" se registra un aumento del uso de las TIC, no solo como disfrute durante el tiempo de ocio sino como una necesidad que intenta satisfacer diferentes fines; desde estar en comunicación con el resto de las personas, a poder seguir cumpliendo con las obligaciones laborales a través de teletrabajo o acceder a toda la información que se va generando en el exterior. Un reciente estudio realizado por Lázaro-Rodríguez, P. y Herrera-Viedma, H. (2020) confirma un elevado 
incremento del volumen de noticias desde el decreto del estado de alarma, llegando casi a triplicar el número de noticias respecto de períodos anteriores a dicho decreto. Considerando así el papel de los medios digitales como protagonista en la difusión de noticias sobre 2019-nCoV y Covid-19. Esto ha provocado que se registrasen incrementos cercanos al $40 \%$ en el tráfico en internet con picos de tráfico récord en alguna ubicación, tal es el caso de Madrid donde se han superado los 500 Gbit por segundo. La Fundación Telefónica en colaboración con otros operadores de telefonía fija y móvil publicaban una nota de prensa solicitando un uso responsable de la red y anunciando que el uso del móvil había aumentado en un $50 \%$ en la voz y un 25 en los datos. En ese mismo comunicado también señalaba que la mensajería instantánea como Whatsapp se había multiplicado por 5 en los primeros días del periodo de confinamiento (Fundación Telefónica, 2020).

Algunos autores que han analizado el impacto de las TIC en la sociedad afirman que la mayoría de los estudios abordan principalmente dos ámbitos, el ámbito laboral y el ámbito educativo (Hernández Prados, López Vicent, \& Sanchez Esteban, 2014), registrándose pocos trabajos en el ámbito personal y/o familiar. Pero se debe destacar que cualquier cambio producido en la sociedad de la información provocará también cambios en el ámbito personal y familiar, con cierta influencia en cambios o adaptaciones del estilo de vida de las personas.

Además de la posible influencia de las TIC en los hábitos sociales y de estilo de vida de los niños, la sociedad española ha estado expuesta a una nueva situación social. El periodo de confinamiento ha privado a muchos niños de continuar con su estilo de vida y hábitos sociales afectando a diferentes esferas de su desarrollo, desde la social a la psíquica. Muchos de ellos pasaron de tener todo el día organizado con diferentes tareas a no poder salir de casa y no saber qué hacer durante tanto tiempo. Por los motivos expuestos, a través de este estudio se pretende conocer cómo han sido los hábitos sociales, de estilo de vida y actividad física de los niños menores de 12 años residentes en España durante el periodo de confinamiento provocado por la pandemia del coronavirus COVID-19 (desde el 15 de marzo hasta el 2 de mayo), mediante un análisis descriptivo y correlacional para cuantificar el número de dispositivos vinculados a las TIC, su tiempo de uso y su correlación con diferentes hábitos y tareas que con normalidad desempeñaban los niños diariamente.

\section{Objetivos}

El objetivo de este trabajo ha sido analizar el número de dispositivos, vinculados a las TIC, que están presentes en los hogares españoles de familias con hijos menores de 12 años, su tiempo de uso por parte de los niños durante el periodo de confinamiento y estudiar su relación con los hábitos sociales, estilo de vida y de actividad física diaria. Se busca conocer si un mayor número de dispositivos tecnológicos o pantallas y/o su tiempo de uso ha influido positiva o negativamente en los períodos dedicados a actividad física, tareas domésticas, lectura, tareas escolares, actividades artísticas o musicales, horas de sueño o juego en familia.

\section{Metodología}

\subsection{Diseño de estudio y participantes}

Para cumplir con los objetivos del presente estudio se realizó una investigación cuantitativa con un diseño no experimental, carácter descriptivo, comparativo y correlacional, con una única medición en un solo grupo. La población objeto de estudio fueron familias españolas con hijos menores de 12 años. De esta población se obtuvo una muestra compuesta por 837 niños españoles, con una edad comprendida entre los 0-12 años $(M=6,22 ;$ D.T.= 3,366). La distribución de la muestra según el sexo de los participantes fue homogénea, representando el $50,2 \%(n=420)$ a los niños y el $49,8 \%$ 
$(n=417)$ a las niñas. Se utilizó un muestreo por conveniencia para seleccionar a los participantes, invitando a participar durante el periodo de confinamiento, a aquellas familias que tenían hijos e hijas en la etapa de infantil y primaria.

\subsection{Instrumento de recogida de datos y variables}

Como instrumento de recogida de datos se utilizó el cuestionario validado sobre Equipamiento y Uso de Tecnologías de Información y Comunicación en los Hogares (TIC-H2019) elaborado por el Instituto Nacional de Estadística (INE) siguiendo las recomendaciones de la Oficina Estadística de la Unión Europea (EUROSTAT) además de la creación de un cuestionario ad hoc de autorregistro a fin de que los progenitores de los niños pudiesen volcar los datos correspondientes a las diferentes variables objeto de estudio.

Las variables registradas en el cuestionario estaban relacionadas con aspectos sociales, culturales y personales como: edad, sexo, número de TIC en el hogar, tiempo de uso, tiempo dedicado a la lectura, juego libre, etc. Para este estudio la investigación se centra en estas dos variables:

1. Recursos y uso de medios tecnológicos. Se registró esta variable, a fin de conocer los medios tecnológicos de los que disponían las familias durante el confinamiento, así como el uso que hacían los niños menos de 12 años. Las familias participantes en el estudio registraron el número de televisores, videoconsolas, ordenadores, teléfonos móviles y tablets de los que disponían en el hogar. Asimismo, también indicaron el tiempo diario (expresado en minutos) que invertían los niños en ellas.

2. Actividades cotidianas en el hogar/estilo de vida. Esta variable hace referencia a todas aquellas acciones que pueden ser desarrolladas en el hogar durante el confinamiento. Para ello, las familias indicaron el tiempo diario (expresado en minutos) dedicado a actividades cotidianas como práctica de actividad física, tareas escolares, tocar instrumentos, realización de actividades artísticas, tareas domésticas, jugar con la familia, lectura y juego libre. De tal forma, la actividad física semanal quedó categorizada en "No realiza", "Realiza 2-3 días a la semana", Realiza 4-5 días a la semana" y "Realiza 6-7 días a la semana".

\subsection{Procedimiento}

Para llegar a la población objeto de investigación se difundió el cuestionario a través de redes sociales (con la siguiente segmentación del público: familias residentes en España con hijos menores de 12 años) y a través del contacto con diversos profesionales de la educación que tuviesen cercanía con un público amplio de familias para asegurar una buena difusión del cuestionario en las diferentes Comunidades Autónomas. El cuestionario se difundió durante 45 días, entre el 23 de marzo y el 6 de mayo del 2020, dentro del periodo de confinamiento establecido por el Gobierno de España Real Decreto 463/2020, de 14 de marzo (Gobierno de España, 2020)

Durante la depuración de la base de datos se eliminaron un total de 76 casos por no encontrarse correctamente cumplimentados o por no pertenecer a la etapa educativa de estudio.

\subsection{Aspectos éticos}

Durante toda la investigación se tuvieron en cuenta los principios éticos reflejados en diferentes documentos y tratados oficiales sobre ética en investigación garantizando así el anonimato de los participantes, la confidencialidad en los datos reflejados en los cuestionarios y otras consideraciones éticas relacionadas con la investigación en educación (American Psychological Association, 2020; Sañudo, 2006). 


\subsection{Análisis de datos}

Para el análisis y tratamiento de los datos se utilizó el software estadístico SPSS 25.0 (IBM Corp, Armonk, NY, USA). En el análisis descriptivo de estudio, se determinaron las características de los participantes mediante la media (M), desviación típica (DT) y frecuencias (\%). La normalidad y homogeneidad de la varianza en las variables, se estipuló con la prueba Kolmogorov-Smirnov. Para establecer las diferencias entre variables se utilizó la prueba ANOVA. Las diferencias entre participantes se determinaron con la prueba Chi-cuadrado de Pearson. Asimismo, se realizó una correlación bivariada de Pearson a nivel de significatividad de $\mathrm{p}<0,05^{*} \mathrm{y} p<0,01^{* *}$. Estas fueron interpretadas como correlaciones débiles $(r<0,29)$, moderadas $(r=0,3-0,49)$ y fuerte $(r=0,5-1)$.

\section{Resultados}

En la tabla 1 se muestran los descriptivos básicos sobre la cantidad y uso de dispositivo vinculados a las TIC en el hogar durante el confinamiento. Estos resultados mostraron que los dispositivos que mayor presencia tienen en el hogar son los televisores $(\mathrm{M}=1,98$; D.T. $=1,02)$, seguidos de ordenadores $(\mathrm{M}=1,81$; D.T. $=1,08)$, tablets $(\mathrm{M}=1,32 ;$ D.T. $=1,00)$ y videoconsolas $(\mathrm{M}=0,88$; D.T. = 1,44). En cuanto al tiempo diario, expresado en minutos, que pasan los jóvenes frente a pantallas digitales durante el confinamiento, se encontraron los valores más elevados en la televisión $(\mathrm{M}=80,38$; D.T. $=61,59)$, seguido del uso de tablets $(\mathrm{M}=28,47$; D.T. $=46,43)$ ordenadores $(\mathrm{M}=$ 18,98; D.T. $=44,56)$, videoconsolas $(M=18,78 ;$ D.T. $=42,86)$ y teléfono móvil $(M=14,58$; D.T. $=$ $39,83)$.

Tabla 1. Descriptivos básicos sobre dispositivos tecnológicos durante el confinamiento

\begin{tabular}{ccccc}
\hline Variables & Mínimo & Máximo & Media & DT \\
\hline Televisores en el hogar & 0 & 6 & 1,98 & 1,02 \\
Videoconsolas en el hogar & 0 & 14 & 0,88 & 1,44 \\
Ordenadores en el hogar & 0 & 7 & 1,81 & 1,08 \\
$\quad$ Tablets en el Hogar & 0 & 7 & 1,32 & 1,00 \\
Teléfono móvil en niños menores & 0 & 1 & 0,09 & 0,28 \\
$\quad$ de 12 años & 0 & 300 & 18,78 & 42,86 \\
Uso diario de Videoconsolas & 0 & 600 & 80,38 & 61,59 \\
Uso diario de la Televisión & 0 & 420 & 18,98 & 44,56 \\
Uso diario de Ordenadores & 0 & 300 & 28,47 & 46,43 \\
$\quad$ Uso diario de Tablets & 0 & 360 & 14,58 & 39,83 \\
\hline Uso diario del Teléfono Móvil & 0 & &
\end{tabular}

Fuente: Elaboración propia

Con relación al acceso a internet se halló que un 99\% de los hogares tenían acceso a internet (70,1\% por fibra óptica, $22,2 \%$ mediante ADSL y un 7,5\% a través de otras conexiones) y solo un $0,1 \%$ no lo tenía.

En la siguiente tabla se puede observar el número de dispositivos tecnológicos presente en los hogares españoles de familias con hijos menores de 12 años estratificados por tres franjas etarias. 
RLCS, Revista Latina de Comunicación Social, 78, 183-204

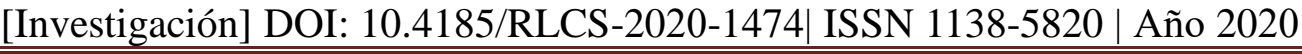

Tabla 2. Descriptivos básicos sobre dispositivos tecnológicos durante el confinamiento clasificados por franjas etarias

\begin{tabular}{|c|c|c|c|c|c|}
\hline \multicolumn{2}{|c|}{ Franja etaria } & $\begin{array}{c}\text { Número de } \\
\text { televisores en el } \\
\text { hogar }\end{array}$ & $\begin{array}{c}\text { Número de } \\
\text { videoconsolas en } \\
\text { el hogar }\end{array}$ & $\begin{array}{l}\text { Número de } \\
\text { ordenadores } \\
\text { en el hogar }\end{array}$ & $\begin{array}{l}\text { Número de } \\
\text { tablets en el } \\
\text { hogar }\end{array}$ \\
\hline \multirow{6}{*}{ Niños de 0-2 años } & $N$ & 202 & 202 & 202 & 202 \\
\hline & Media & 1,78 & ,59 & 1,70 & 1,12 \\
\hline & DT & 0,926 & 1,067 & 0,927 & 0,955 \\
\hline & Mínimo & 0 & 0 & 0 & 0 \\
\hline & Máximo & 5 & 12 & 7 & 7 \\
\hline & $\begin{array}{l}\% \text { de Niños que } \\
\text { al menos tienen } \\
\text { una unidad }\end{array}$ & $98,5 \%$ & $42,1 \%$ & $97,5 \%$ & $78,5 \%$ \\
\hline \multirow{6}{*}{ Niños de 3 a 6 años } & $N$ & 260 & 260 & 260 & 260 \\
\hline & Media & 1,96 & 0,67 & 1,65 & 1,23 \\
\hline & DT & 1,005 & 1,220 & 0,973 & 867 \\
\hline & Mínimo & 1 & 0 & 0 & 0 \\
\hline & Máximo & 6 & 11 & 6 & 4 \\
\hline & $\begin{array}{l}\% \text { de Niños que } \\
\text { al menos tienen } \\
\text { una unidad }\end{array}$ & $100 \%$ & $43,5 \%$ & $94,6 \%$ & $81,2 \%$ \\
\hline \multirow{7}{*}{$\begin{array}{l}\text { Niños de } 7 \text { a } 12 \\
\text { años }\end{array}$} & $N$ & 375 & 375 & 375 & 375 \\
\hline & Media & 2,11 & 1,19 & 1,99 & 1,48 \\
\hline & DT & 1,062 & 1,694 & 1,200 & 1,094 \\
\hline & Mínimo & 0 & 0 & 0 & 0 \\
\hline & Máximo & 6 & 14 & 7 & 7 \\
\hline & $\begin{array}{l}\% \text { de Niños que } \\
\text { al menos tienen } \\
\text { una unidad }\end{array}$ & $99,5 \%$ & $69,1 \%$ & $98,4 \%$ & $85,1 \%$ \\
\hline & & $p=0,001$ & $p=0,000$ & $\mathrm{p}=0,000$ & $p=0,000$ \\
\hline
\end{tabular}

Fuente: Elaboración propia

En la tabla 3 se realizan un análisis descriptivo del porcentaje de niños clasificados en 3 intervalos de edades que realiza la cantidad mínima de actividad física diaria recomendada por diferentes organismos internacionales y los valores medios de actividad física al día durante el periodo de confinamiento.

Tabla 3. Descriptivos básicos sobre niveles de actividad física durante el confinamiento clasificados por franjas etarias

\begin{tabular}{|c|c|c|c|}
\hline \multicolumn{2}{|c|}{ Franja etaria } & $\begin{array}{l}\text { \% que realiza actividad física } \\
\text { diaria (mínimo } 60 \text { minutos) }\end{array}$ & $\begin{array}{c}\text { Tiempo medio al día de } \\
\text { actividad física }\end{array}$ \\
\hline \multirow[b]{2}{*}{ Niños de 0-2 años } & $N$ & 202 & 202 \\
\hline & Valor & $12,4 \%$ & $\mathrm{M}=30,86 \mathrm{DT}=42,59$ \\
\hline \multirow[b]{2}{*}{ Niños de 3 a 6 años } & $N$ & 260 & 260 \\
\hline & Valor & $15,0 \%$ & $\mathrm{M}=37,26 \mathrm{DT}=36,01$ \\
\hline & $N$ & 375 & 375 \\
\hline 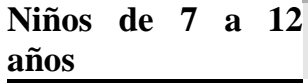 & Valor & $12,0 \%$ & $\mathrm{M}=38,95 \mathrm{DT}=30,25$ \\
\hline
\end{tabular}

Fuente: Elaboración propia 
En la siguiente tabla se establecen las relaciones entre el número y uso de dispositivos tecnológicos de pantalla en función de la práctica de actividad física durante el confinamiento. Entre estas asociaciones se encontraron resultados estadísticamente significativos $(p<0,05)$. Una mayor frecuencia semanal de práctica de actividad física (6-7 días a la semana) se relacionó con una mayor presencia de ordenadores $(\mathrm{M}=2,07$; D.T. $=1,75)$ y tablets $(\mathrm{M}=1,58$; D.T. $=1,18)$ en el hogar, frente a los que no practican $(\mathrm{M}=1,63 ;$ D.T. $=0,95$ y $\mathrm{M}=1,17$; D.T. $=0,90)$. En este sentido, los jóvenes que pasaron más tiempo frente a las pantallas de las tablets $(\mathrm{M}=37,51$; D.T. $=52,62)$, realizaban actividad física 4-5 días a la semana. Sin embargo, aquellos que manifestaron que no practicaban actividad semanal, mostraron los valores más altos en el uso de videoconsolas $(\mathrm{M}=$ 26,48; D.T. $=55,82)$, televisión $(M=84,63$; D.T. $=70,68)$, ordenadores $(M=84,63$; D.T. $=70,68)$ y teléfono móvil $(\mathrm{M}=18,43 ;$ D.T. $=47,69)$.

Tabla 4. Variables tecnológicas en función de la práctica de actividad física semanal durante el confinamiento

\begin{tabular}{|c|c|c|c|c|c|c|}
\hline Variable & AF semanal & $\mathbf{M}$ & DT & Error Std. & $\mathbf{F}$ & $\mathbf{X}^{2}$ \\
\hline \multirow{4}{*}{$\begin{array}{c}\text { Televisores en el } \\
\text { hogar }\end{array}$} & No Practica & 1,92 & 0,97 & 0,05 & \multirow{4}{*}{1,517} & \multirow{4}{*}{0,209} \\
\hline & 2-3 días & 2,01 & 1,08 & 0,06 & & \\
\hline & 4-5 días & 2,10 & 0,95 & 0,07 & & \\
\hline & 6-7 días & 1,89 & 1,05 & 0,10 & & \\
\hline \multirow{4}{*}{$\begin{array}{c}\text { Videoconsolas en el } \\
\text { hogar }\end{array}$} & No Practica & 1,00 & 1,70 & 0,10 & \multirow{4}{*}{1,004} & \multirow{4}{*}{0,390} \\
\hline & 2-3 días & 0,83 & 1,52 & 0,09 & & \\
\hline & 4-5 días & 0,82 & 1,05 & 0,08 & & \\
\hline & 6-7 días & 0,80 & 0,91 & 0,08 & & \\
\hline \multirow{4}{*}{$\begin{array}{c}\text { Ordenadores en el } \\
\text { hogar }\end{array}$} & No Practica & 1,63 & 0,95 & 0,05 & \multirow{4}{*}{7,600} & \multirow{4}{*}{$\mathbf{0 , 0 0 0}$} \\
\hline & 2-3 días & 1,77 & 0,98 & 0,05 & & \\
\hline & 4-5 días & 2,04 & 1,18 & 0,09 & & \\
\hline & 6-7 días & 2,07 & 1,35 & 0,12 & & \\
\hline \multirow{4}{*}{ Tablets en el hogar } & No Practica & 1,17 & 0,90 & 0,05 & \multirow{4}{*}{6,202} & \multirow{4}{*}{$\mathbf{0 , 0 0 0}$} \\
\hline & 2-3 días & 1,27 & 0,86 & 0,05 & & \\
\hline & 4-5 días & 1,48 & 1,19 & 0,09 & & \\
\hline & 6-7 días & 1,58 & 1,18 & 0,11 & & \\
\hline \multirow{4}{*}{$\begin{array}{c}\text { Tiempo diario de } \\
\text { uso de } \\
\text { videoconsolas }\end{array}$} & No Practica & 26,48 & 55,82 & 4,35 & \multirow{4}{*}{2,751} & \multirow{4}{*}{$\mathbf{0 , 0 4 4}$} \\
\hline & 2-3 días & 15,14 & 37,69 & 2,28 & & \\
\hline & 4-5 días & 18,78 & 40,78 & 2,39 & & \\
\hline & 6-7 días & 16,34 & 36,69 & 3,51 & & \\
\hline \multirow{4}{*}{$\begin{array}{c}\text { Tiempo diario de } \\
\text { uso de la televisión }\end{array}$} & No Practica & 84,63 & 70,68 & 4,14 & \multirow{4}{*}{5,132} & \multirow{4}{*}{$\mathbf{0 , 0 1 0}$} \\
\hline & 2-3 días & 83,31 & 63,04 & 3,81 & & \\
\hline & 4-5 días & 72,18 & 42,44 & 3,31 & & \\
\hline & 6-7 días & 74,03 & 54,83 & 5,25 & & \\
\hline \multirow{4}{*}{$\begin{array}{l}\text { Tiempo diario de uso } \\
\text { de ordenadores }\end{array}$} & No Practica & 84,63 & 70,68 & 4,14 & \multirow{4}{*}{2,901} & \multirow{4}{*}{0,041} \\
\hline & 2-3 días & 83,31 & 63,04 & 3,81 & & \\
\hline & 4-5 días & 72,18 & 42,44 & 3,31 & & \\
\hline & 6-7 días & 74,03 & 54,83 & 5,25 & & \\
\hline \multirow{4}{*}{$\begin{array}{c}\text { Tiempo diario de uso } \\
\text { de Tablets }\end{array}$} & No Practica & 28,89 & 53,51 & 3,13 & \multirow{4}{*}{3,191} & \multirow{4}{*}{$\mathbf{0 , 0 2 3}$} \\
\hline & 2-3 días & 23,74 & 35,94 & 2,17 & & \\
\hline & 4-5 días & 37,51 & 52,63 & 4,11 & & \\
\hline & 6-7 días & 25,56 & 37,08 & 3,55 & & \\
\hline \multirow{4}{*}{$\begin{array}{l}\text { Tiempo diario de uso } \\
\text { de teléfono móvil }\end{array}$} & No Practica & 18,43 & 47,69 & 2,79 & \multirow{4}{*}{4,963} & \multirow{4}{*}{0,018} \\
\hline & 2-3 días & 14,75 & 39,03 & 2,36 & & \\
\hline & 4-5 días & 10,52 & 25,52 & 1,99 & & \\
\hline & 6-7 días & 10,00 & 35,58 & 3,40 & & \\
\hline
\end{tabular}

Fuente: Elaboración propia 
En la siguiente tabla, se muestran las correlaciones entre recursos tecnológicos y uso diario de pantallas digitales en relación con los hábitos sociales y estilo de vida de los niños adoptado en el hogar durante el confinamiento. El tiempo diario empleado a realizar actividad física (TDAF) se relacionó directamente con el número de ordenadores $\left(\mathrm{r}=0,150^{*}\right)$ y tablets $\left(\mathrm{r}=0,269^{* *}\right)$, aunque lo hizo indirectamente con el número de videoconsolas $\left(\mathrm{r}=-0,232^{* *}\right)$ y el uso diario de teléfonos móviles $\left(\mathrm{r}=-0,270^{* *}\right)$. El tiempo dedicado a las tareas escolares (TDTE), se asoció directamente con el número de televisores $\left(r=0,227^{* *}\right)$, videoconsolas $\left(r=0,178^{*}\right)$ y tablets $\left(r=0,194^{* *}\right)$, siendo esa asociación moderada con el uso diario de videoconsolas $\left(\mathrm{r}=0,346^{* *}\right)$, televisores $\left(\mathrm{r}=0,344^{* *}\right)$, ordenadores $\left(\mathrm{r}=0,421^{* *}\right)$, tablets $\left(\mathrm{r}=0,317^{* *}\right)$ y móviles $\left(\mathrm{r}=0,322^{* *}\right)$. El tiempo dedicado a actividades artísticas (TDART) se asoció positivamente con el uso diario de la televisión ( $\mathrm{r}=$ $\left.0,240^{* *}\right)$, aunque lo hizo negativamente con el número de televisiones $\left(\mathrm{r}=-0,189^{* * *}\right)$, videoconsolas $(\mathrm{r}$ $\left.=0,191^{* *}\right)$, ordenadores $\left(\mathrm{r}=-0,206^{* * *}\right)$ y uso diario de videoconsolas $\left(\mathrm{r}=-0,245^{* *}\right)$. Las tareas domésticas (TDDOM) se relacionaron directamente con el uso diario de videoconsolas $\left(\mathrm{r}=0,206^{* *}\right)$, televisión $\left(\mathrm{r}=0,246^{* *}\right)$ y tablets $\left(0,189^{*}\right)$, siendo moderado con el uso de ordenadores $\left(\mathrm{r}=0,307^{* * *}\right)$ y móviles $\left(\mathrm{r}=0,317^{* *}\right)$.

En este sentido, el número de televisores $\left(\mathrm{r}=-0,214^{* *}\right)$ y videoconsolas $\left(\mathrm{r}=-0,172^{*}\right)$, así como el uso diario de videoconsolas $\left(\mathrm{r}=-0,267^{* *}\right)$, ordenadores $\left(\mathrm{r}=-0,269^{* *}\right)$, tablets $\left(\mathrm{r}=-0,181^{*}\right)$ y móviles $(\mathrm{r}=$ $-0,172^{*}$ ) se relacionó indirectamente con el tiempo dedicado a jugar entre la familia (TDFAM), aunque ver la televisión $\left(\mathrm{r}=0,170^{*}\right)$ lo hizo directamente. El tiempo dedicado a la lectura (TDL) se asoció negativamente con el número de televisores $\left(\mathrm{r}=-0,188^{*}\right)$, sin embargo, presentó una correlación moderada y positiva con el uso de ordenadores $\left(\mathrm{r}=0,332^{* *}\right)$. Asimismo, el tiempo dedicado al juego libre de los niños (TDJL) se relacionó negativamente con el número de televisores $\left(\mathrm{r}=-0,200^{* * *}\right)$, videoconsolas $\left(\mathrm{r}=-0,193^{* *}\right)$ y su uso $\left(\mathrm{r}=-0,262^{* * *}\right)$, uso de ordenadores $\left(\mathrm{r}=-0,278^{* * *}\right)$ y teléfonos móviles $\left(\mathrm{r}=-0,188^{*}\right)$. 
RLCS, Revista Latina de Comunicación Social, 78, 183-204

[Investigación] DOI: 10.4185/RLCS-2020-1474| ISSN 1138-5820| Año 2020

Tabla 5. Correlación de los dispositivos tecnológicos y uso diario de pantallas digitales en función de aspectos cotidianos en el hogar durante el confinamiento.

\begin{tabular}{|c|c|c|c|c|c|c|c|c|c|c|c|c|c|c|c|c|}
\hline & NVC & NPC & NTB & UDVC & UDTV & UDPC & UDTB & UDTM & TDAF & TDTE & TDINS & TDART & TDDOM & TDFAM & TDL & TDJL \\
\hline NTV & $0,365^{* *}$ & 0,020 & $0,209^{* *}$ & $0,280^{* * *}$ & $0,204^{* *}$ & 0,037 & $0,231^{* *}$ & $0,209^{* *}$ & $\overline{0,041}$ & $0,227^{* * *}$ & $-0,056$ & $-0,189^{* *}$ & $-0,031$ & $-0,214^{* *}$ & $-0,188^{*}$ & $-0,200^{* *}$ \\
\hline NVC & 1 & $0,237^{* * *}$ & $0,250^{* *}$ & $0,375^{* *}$ & $-0,014$ & $0,168^{*}$ & $0,190^{* *}$ & $-0,001$ & $0, \overline{232}^{* * *}$ & $0,178^{*}$ & 0,023 & $-0,191^{* *}$ & $-0,016$ & $-0,172^{*}$ & $\mathbf{0 , 0 3 8}$ & $-0,193^{* *}$ \\
\hline NPC & & 1 & $0,470^{* *}$ & 0,067 & $-0,178^{*}$ & $0,358^{* *}$ & 0,043 & $-0,020$ & $0,150 *$ & 0,058 & 0,065 & $-0,206^{* * *}$ & $-\mathbf{0 , 0 3 3}$ & $-0,053$ & $\mathbf{0 , 0 3 4}$ & $-0,014$ \\
\hline NTB & & & 1 & $0,201^{* *}$ & $-0,055$ & 0,041 & $0,376^{* *}$ & $0, \overline{-}^{-} 7^{* *}$ & $0,269^{* * *}$ & $0,194^{* *}$ & 0,018 & $-0,047$ & $\mathbf{0 , 0 3 5}$ & $-0,035$ & $\mathbf{0 , 0 5 9}$ & $-0,049$ \\
\hline $\begin{array}{l}\text { UDVC } \\
\text { UDTV }\end{array}$ & & & & 1 & $\begin{array}{c}0,054 \\
1\end{array}$ & $\begin{array}{l}0,265^{* *} \\
0,198^{* *}\end{array}$ & $\begin{array}{c}0,221^{* *} \\
0,176^{*}\end{array}$ & $\begin{array}{c}0,187^{*} \\
0,321^{* *}\end{array}$ & $\begin{array}{r}-0,006 \\
-0,015\end{array}$ & 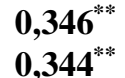 & $\begin{array}{c}-0,011 \\
0,012\end{array}$ & $\begin{array}{c}-0,245^{* * *} \\
0,240^{* *}\end{array}$ & $\begin{array}{l}\mathbf{0 , 2 0 6} \\
\mathbf{0 , 2 4 6}^{* * *}\end{array}$ & $\begin{array}{c}-0,267^{* *} \\
0,170^{*}\end{array}$ & $\begin{array}{l}\mathbf{0 , 0 4 3} \\
\mathbf{0 , 0 4 4}\end{array}$ & $\begin{array}{c}-0,262^{* * *} \\
0,039\end{array}$ \\
\hline UDPC & & & & & & 1 & 0,017 & $0,271^{* *}$ & 0,006 & $0,421^{* *}$ & 0,052 & $-0,031$ & $\mathbf{0 , 3 0 7 ^ { * * }}$ & $-0,269^{* *}$ & $\mathbf{0 , 3 3 2}{ }^{* *}$ & $-0,278^{* *}$ \\
\hline UDTB & & & & & & & 1 & $-0,057$ & $\mathbf{0 , 0 3 1}$ & $\mathbf{0 , 3 1 7} 7^{* * *}$ & 0,002 & $-0,010$ & $\mathbf{0 , 1 8 9}{ }^{*}$ & $-0,181^{*}$ & $-0,024$ & $-0,015$ \\
\hline UDTM & & & & & & & & 1 & $0, \overline{-} 70^{* * *}$ & $0,322^{* *}$ & $-0,003$ & $-0,006$ & $\mathbf{0 , 3 1 7 ^ { * * }}$ & $-0,172^{*}$ & 0,034 & $-0,188^{*}$ \\
\hline TDAF & & & & & & & & & 1 & $0,220^{* *}$ & 0,038 & $0,241^{* *}$ & $0,246^{* *}$ & 0,068 & $0,251^{* *}$ & $0,198^{* *}$ \\
\hline TDTE & & & & & & & & & & 1 & 0,045 & $0,186^{*}$ & $0,334^{* *}$ & $-0,284^{* *}$ & $0,381^{* * *}$ & $-0,196^{* *}$ \\
\hline TDINS & & & & & & & & & & & 1 & 0,053 & $0,233^{* *}$ & 0,046 & $0,190^{* *}$ & $-0,015$ \\
\hline TDART & & & & & & & & & & & & 1 & $0,399^{* *}$ & $0,351^{* * *}$ & $0,316^{* * *}$ & $0,326^{* * *}$ \\
\hline TDDOM & & & & & & & & & & & & & 1 & $0,194^{* * *}$ & $0,410^{* * *}$ & 0,012 \\
\hline TDFAM & & & & & & & & & & & & & & 1 & $0,224^{* *}$ & $0,473^{* *}$ \\
\hline TDL & & & & & & & & & & & & & & & 1 & $0,174^{*}$ \\
\hline TDJL & & & & & & & & & & & & & & & & 1 \\
\hline
\end{tabular}

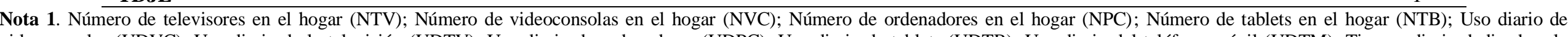

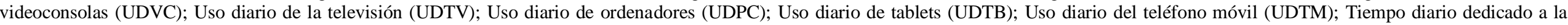

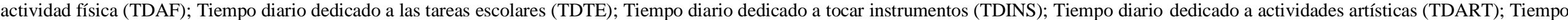
diario dedicado a tareas domésticas (TDDOM); Tiempo diario dedicado a jugar con la familia (TDFAM); Tiempo diario dedicado a la lectura (TDL); Tiempo diario dedicado al juego libre (TDJL);

Nota 2. Correlación bivariada de Pearson a nivel de significatividad de $\mathrm{p}<0,05(*)$ y $\mathrm{p}<0,01(* *)$

Fuente: Elaboración propia 


\section{Discusión}

El presente trabajo analizó el número de TIC y otros dispositivos tecnológicos de pantalla presentes en los hogares españoles con hijos menores de 12 años y el tiempo de uso dedicado por los menores a cada uno de estos dispositivos durante el periodo de confinamiento provocado por la COVID-19. Además, se han correlacionado estos dos aspectos con diferentes variables vinculadas a los hábitos sociales de los niños, estilo de vida y práctica de actividad física.

Los resultados muestran que las familias españolas con hijos menores de 12 años participantes en esta investigación han pasado el periodo de confinamiento con la presencia de un número importante de TIC en sus hogares. Este dato confirma los resultados del estudio de la Asociación para la Investigación de Medios de Comunicación (AIMC) que percibe que en los hogares de los niños españoles menores de 13 años existe un alto nivel de equipamiento tecnológico, constatando una media de casi 7 dispositivos tecnológicos y, de éstos, los más pequeños de la casa utilizan una media de 4 (Asociación para la Investigación de Medios de Comunicación, 2018). Los televisores fueron los dispositivos más frecuentes en los hogares de familias con hijos menores de 12 años, con una media cercana a dos unidades por familia. Los resultados de la última encuesta del INE (2019) muestran valores similares al señalar que el televisor es el dispositivo más presente en los hogares españoles con un porcentaje del 99,5\% en viviendas de parejas con hijos. También el reciente estudio elaborado por AIMC sobre una muestra de 5190 niños españoles señala que la televisión es el dispositivo más utilizado por ellos para ver los contenidos televisivos (91\%) (Asociación para la Investigación de Medios de Comunicación, 2018). Otros trabajos también han confirmado la presencia de un importante contexto multipantalla en los hogares de niños españoles calificando a la población infantil como una población hiperconectada a partir de los 7 años, intensificándose a partir de los 10 años y señalando a los padres como los principales responsables del uso de pantallas en niños (Torrecillas Lacave, Vázquez Barrio, Suárez Álvarez, Suárez Álvarez, \& Fernández Martínez, 2020).

Hay que destacar que en el presente estudio el $99 \%$ de los hogares tenían acceso a internet, una cifra similar a la recogida por el INE (2019) que ubica en un 99,8\% los hogares de parejas con hijos con acceso a internet en España. En este último trabajo se indica que las viviendas que no disponen de internet señalan como principales motivos de tal situación los siguientes: porque no necesitan internet $(75,5 \%)$, la falta de conocimientos para usarlo $(51,3 \%)$ y los altos costes del equipo (28,0\%).

Los ordenadores y las tablets constituyeron el segundo grupo de dispositivos más presentes en los hogares con valores medios de 1,81 y 1,32 respectivamente. En la encuesta del INE (2019) se constata que el $93,1 \%$ de las familias con hijos tienen estos dispositivos en su hogar. Las videoconsolas fueron el dispositivo electrónico de pantalla con menor presencia en los hogares de las familias con menores de 12 años ubicando la media en 0,88 por hogar llegando. Sin embargo, en algún caso, este dispositivo alcanzó el máximo valor de todos los dispositivos analizados, llegando a existir en un mismo hogar un total de 14 videoconsolas. En el estudio de AIMC (Asociación para la Investigación de Medios de Comunicación, 2018) se observó que un 39\% de los niños entrevistados tenían en propiedad una Tablet, un 34,7\% una videoconsola y un 27,1\% dispone de un smartphone. En nuestro trabajo se confirma que en la franja etaria de 7 a 12 años el 69,1\% de los niños tienen al menos una videoconsola en su hogar y un $85,1 \%$ una Tablet.

En relación con el estilo de vida de los niños durante el periodo de confinamiento y su correlación con el número de dispositivos tecnológicos y uso de estos se debe comentar primeramente que la limitación de sus posibilidades de acción y el espacio reducido de muchos hogares provocaron cambios en el estilo de vida de todos los miembros de la familia. En cuanto a los niveles de actividad 
física en niños menores de 12 años recomendados por diferentes organismos internacionales (Piercy et al., 2018; OMS, 2019) han constituido un verdadero reto para muchas familias. De por sí, los niveles habituales de práctica de actividad física en los niños españoles no son relativamente altos, registrando diversos trabajos porcentajes del $70 \%$ de los niños y $88 \%$ de las niñas de entre 2 y 10 años que no cumplen con las recomendaciones de 60 minutos de actividad física diaria (Fundación para la Investigación Nutricional, 2016). En otro trabajo realizado en una muestra de 3598 niños de entre 10 y 14 años se constató que el 64,3\% de los estudiantes no cumplían las recomendaciones de actividad física (Herazo-Beltran et al., 2019). El periodo de confinamiento supuso un mayor obstáculo para la práctica de actividad física de los niños, verificando que solamente un porcentaje inferior al 13,1 \% de los niños cumplieron con las recomendaciones de actividad física diaria, lo que implica que un $86,9 \%$ no cumplieron las recomendaciones, una cifra más elevada que la confirmada por diversos estudios sobre los niveles de práctica habitual de actividad física en niños.

Pero qué influencia pudo tener en estos niveles la presencia y uso de los diferentes dispositivos tecnológicos hallados en el hogar durante el periodo de confinamiento. En este sentido se ha encontrado una correlación entre el tiempo de uso de diferentes dispositivos y la ausencia de actividad física. Los progenitores que manifestaron que sus hijos no realizaban nada de actividad física durante el periodo de confinamiento señalaron valores más altos en el uso de videoconsolas (M $=26,48 ;$ D.T. $=55,82)$, televisión $(\mathrm{M}=84,63 ;$ D.T. $=70,68)$, ordenadores $(\mathrm{M}=84,63 ;$ D.T. $=70,68)$ y teléfono móvil $(\mathrm{M}=18,43 ;$ D.T. $=47,69)$. En otros países también se ha constatado niveles bajos de actividad física y gran cantidad de tiempo frente a diferentes pantallas (Maher et al., 2019). Un estudio realizado en una muestra de 267 niños en su primer año de escuela primeria confirma la necesidad de fomentar estilos de vida saludables que aborden integralmente la nutrición, el tiempo dedicado a la actividad física y los videojuegos, dado que sus autores encontraron probabilidades más altas de una mala adherencia a una dieta saludable en niños que jugaban a videojuegos más de una hora al día (Buja, Grotto, Brocadello, Sperotto, \& Baldo, 2020).

Por otro lado, el tiempo dedicado a las tareas escolares durante el periodo de confinamiento se asoció directamente con el número de televisores, videoconsolas y tablets, siendo esa asociación moderada con el uso diario de videoconsolas, televisores, ordenadores, tablets y móviles. El tiempo dedicado a actividades artísticas, como pintar, dibujar, fotografiar, etc. se asoció positivamente con el uso diario de la televisión, aunque lo hizo negativamente con el número de televisiones, videoconsolas, ordenadores y uso diario de videoconsolas. Las tareas domésticas se relacionaron directamente con el uso diario de videoconsolas, televisión y tablets, siendo moderado con el uso de ordenadores y móviles.

Por último, el tiempo dedicado a jugar en familia se asoció con el tiempo dedicado a ver la televisión e indirectamente con el número de televisores, el uso diario de la videoconsola, ordenador, tablet y móvil. En un reciente estudio realizado en 946 jóvenes de 11 a 18 años de edad las correlaciones preliminares indicaron que puntuaciones más altas en ansiedad, impulsividad, hostilidad y déficit de habilidades sociales y puntuaciones más bajas en el funcionamiento familiar (relación interpersonal) y más tiempo dedicado a los videojuegos se asociaron con el trastorno de adición a los videojuegos (Fumero et al., 2020).

El tiempo dedicado a la lectura se asoció negativamente con el número de televisores, sin embargo, presentó una correlación moderada y positiva con el uso de ordenadores. Asimismo, el tiempo dedicado al juego libre de los niños se relacionó negativamente con el número de televisores, videoconsolas y su uso, uso de ordenadores y teléfonos móviles. Diversos hábitos o actividades que debieran estar más presentes en el estilo de vida de los niños son eclipsados por el tiempo de uso de las videoconsolas. Esto coincide con los resultados de otros autores (Chacón Cuberos, 2018) que 
destacan que la mayoría de los preadolescentes poseía alguna videoconsola y más de la mitad jugaban al menos un día a la semana. Se concluía que un $15 \%$ de los escolares señaló que reemplazaba otras actividades por jugar a la videoconsola y un tercio que se sentía de mal humor si no lo hacía. La riqueza de un estilo de vida saludable para un niño se encuentra en la posibilidad de realizar diversas actividades que contribuyan a un mejor desarrollo de sus habilidades y capacidades, evitando eclipsar algunas de estas con el sobreuso del uso de determinados dispositivos tecnológicos de pantalla. En este sentido, algunos autores recomienda además de enseñar a los progenitores sobre los posibles peligros de la red educar a estos en el uso de las TIC a fin de poder establecer una comunicación asertiva con sus hijos (Condeza, Herrada-Hidalgo, \& Barros-Friz, 2019).

\section{Conclusiones}

Se concluye que durante el periodo de confinamiento los niños españoles menores de 12 años hicieron un gran uso de las TIC debido principalmente a un mayor tiempo en casa y especialmente a una importante cantidad de dispositivos tecnológicos de pantalla en la gran mayoría de los hogares. El televisor fue el dispositivo más presente en los hogares y también el más utilizado, llegando a cuadruplicar el tiempo invertido en él frente a otros dispositivos, según los estudios consultados porque se comparte con toda la familia, mientras el resto de los dispositivos son de uso más individual. Los ordenadores y las tablets también tuvieron un importante protagonismo, siendo los ordenadores los segundos dispositivos más presentes en los hogares y las tablets el segundo dispositivo más usado por los niños durante el confinamiento. Sumando el tiempo dedicado a los diferentes dispositivos tecnológicos analizados en este trabajo la media de uso de las TIC se situó en valores cercanos a las 3 horas diarias en los menores de 12 años.

En relación con la posible asociación entre el uso y número de las TIC y el estilo de vida de los niños hay que destacar que se encontraron diferentes asociaciones estadísticamente significativas entre diversos hábitos sociales, actividades diarias de los niños y el tiempo de uso o presencia de dispositivos en el hogar. El tiempo dedicado a los videojuegos se relacionó negativamente con el de actividad física, el de aprender a tocar un instrumento, el dedicado a actividades artísticas, a la familia y al juego libre. El tiempo destinado al juego libre, algo que es importante en niños de 0 a 12 años, se relacionó negativamente con un número destacado de dispositivos, entre los que están los televisores, videoconsolas, ordenadores y teléfonos móviles. Por otro lado, durante el periodo de confinamiento un porcentaje muy alto de niños, aproximadamente el $85 \%$, no pudo cumplir con la cantidad mínima de actividad física recomendada por organismos internacionales para un estilo de vida saludable, con las posibles consecuencias que esto puede tener para su salud y bienestar físico, social, afectivo y emocional. La situación especial de confinamiento provocada por la pandemia COVID-19 supuso una importante alteración de las rutinas de los niños españoles.

\section{Recomendaciones y perspectivas de futuro}

En este estudio se ha realizado un análisis principalmente descriptivo y correlacional sobre el número de dispositivos electrónicos y tiempo de uso por parte de los niños menores de 12 años durante el periodo de confinamiento. De cara a futuros trabajos sería recomendable analizar el posible uso educativo vinculado a las TIC presentes en los hogares de los niños, analizando sí los progenitores tienen formación suficiente para que, en el caso de nuevos periodos de confinamiento, puedan explotar al máximo las posibilidades educativas que ofrecen los diferentes dispositivos electrónicos. En caso de detectarse una formación insuficiente de los progenitores hacia el manejo y uso de las TIC desde una perspectiva educativa sería recomendable dar formación a las familias sobre su uso para que puedan atender los requerimientos mínimos de las tareas escolares que implica la educación no presencial. Ante el hallazgo de estos resultados sería recomendable establecer pautas de actuación y dar formación a las familias sobre uso de las TIC en niños para poder gestionar en un futuro 
situaciones como esta, evitando, en la medida de lo posible, alterar el día a día de los niños sin abusar del uso de determinados dispositivos tecnológicos de pantalla que pudiese ir en detrimento de la realización de otras actividades sociales, culturales, saludables y/o educativas que contribuyan al desarrollo integral del niño.

\section{Bibliográfía}

American Psychological Association (2020). Publication manual of the american psychological association ( $7^{\mathrm{a}}$ ed.). Estados Unidos: American Psychological Association. https://doi.org/10.1037/0000165-000

Asociación para la Investigación de Medios de Comunicación (2018). Informe estudio AIMC en niños. Madrid: AIMC.

Barja-Fernández, S., Pino-Juste, M. R., Portela-Pino, I., \& Leis-Trabazo, M. R. (2020). Evaluación de los hábitos de alimentación y actividad física en escolares gallegos. Nutrición Hospitalaria: Órgano Oficial de la Sociedad Española de Nutrición Parenteral y Enteral, 37(1), 93-100. https://doi.org/10.20960/nh.02668

Barker, J. E., Semenov, A. D., Michaelson, L., Provan, L. S., Snyder, H. R., \& Munakata, Y. (2014). Less-structured time in children's daily lives predicts self-directed executive functioning. Frontiers in Psychology, 5(593), 1-16. https://doi.org/10.3389/fpsyg.2014.00593

Boente-Antela, B., Leirós-Rodríguez, R., \& García-Soidán, J. L. (2020). ¿Los menores españoles, en su tiempo libre, prefieren dispositivos electrónicos o actividad física? Sportis: Revista TécnicoCientífica del Deporte Escolar, Educación Física y Psicomotricidad, 6(2), 347-364. https://doi.org/10.17979/sportis.2020.6.2.6160

Buja, A., Grotto, G., Brocadello, F., Sperotto, M., \& Baldo, V. (2020). Primary school children and nutrition: Lifestyles and behavioral traits associated with a poor-to-moderate adherence to the mediterranean diet. A cross-sectional study. European Journal of Pediatrics, 5(179), 827-834. https://doi.org/10.1007/s00431-020-03577-9

Capistrán-Gracia, R. W. (2020). Educación musical y bienestar psicológico en los niños y adolescentes en etapa temprana de la "Ciudad de los niños de Aguascalientes". ArtsEduca, 25, pp. 123-148 https://doi.org/10.6035/artseduca.2020.25.9

Chacón-Cuberos, R. (2018). Caracterización de indicadores deportivos, psicosociales y de ocio digital en la comunidad educativa de granada: Construyendo una educación físico-saludable integral mediante videojuegos activos.(tesis doctoral). Universidad de Granada., Granada. https://doi.org/10.7203/relieve.24.1.12614

Chamarro-Lusar, A., Carbonell, X., Manresa, J. M., Muñoz-Miralles, R., Ortega-González, R., López-Morrón, M. R., Batalla-Martínez, C.; Torán-Montserrat, P. (2014). El cuestionario de experiencias relacionadas con los videojuegos (CERV): Un instrumento para detectar el uso problemático de videojuegos en adolescentes españoles. Adicciones: Revista de Socidrogalcohol, 26 (4), 303-311. https://doi.org/10.20882/adicciones.31

Christiansen, N. B. (2018). LEGO play well report 2018. Estados Unidos: The LEGO Foundation.

Condeza, R., Herrada-Hidalgo, N., \& Barros-Friz, C. (2019). Nuevos roles parentales de mediación: Percepciones de los padres sobre la relación de sus hijos con múltiples pantallas. El Profesional de la Información, 28(4). https://doi.org/10.3145/epi.2019.jul.02 
RLCS, Revista Latina de Comunicación Social, 78, 183-204

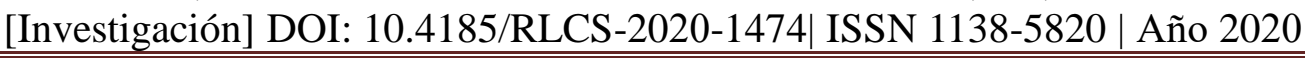

Cuesta-Cambra, U., Cuesta-Díaz, V., Martínez, L., \& Niño-González, J. I. (2020). Smartphone: En comunicación, algo más que una adicción. Revista Latina de Comunicación Social, 75, pp. 367381 https://doi.org/10.4185/rlcs-2020-1431

Fiz-Poveda, M. R., Olea-Aisa, M. J., Goicoechea-Tabar, M. J., \& Ibiricu-Díaz, O. (2000). Los hábitos de lectura y su relación con otras variables. Huarte de San Juan. Filología y Didáctica de la Lengua, 5, 7-31. https://hdl.handle.net/2454/29007

Fumero, A., Marrero, R. J., Bethencourt, J. M., \& Peñate, W. (2020). Risk factors of internet gaming disorder symptoms in spanish adolescents. Computers in Human Behavior, 111. https://doi.org/10.1016/j.chb.2020.106416

Fundación para la Investigación Nutricional (2016). Informe 2016: Actividad física en niños y adolescentes en España. Madrid: Fundación para la Investigación Nutricional. https://doi.org/10.13184/eidon.39.2013.86-88

Fundación Telefónica. (2020). Los operadores aconsejan un uso racional y responsable de las redes de telecomunicaciones para afrontar los incrementos de tráfico (comunicado de prensa).

García, S. (2017). Actitudes de género sobre las responsabilidades del cuidado de los hijos y tareas domésticas de hombres y mujeres en parejas de doble ingreso con hijos menores en nuevo león. Perspectivas Sociales, 19(1), 89-113. https://doi.org/10.22201/crim.unam000001c.2017.c32

Gil-Quintana, J., \& Cano-Alfaro, A. (2020). Inclusión digital: Un reto para la organización, planificación y didáctica escolar. Mediterranean Journal of Communication, 11(1), 51-60. https://doi.org/10.14198/medcom2020.11.1.6

Ginsburg, K. R., Committee on Communications \& Committee on Psychosocial Aspects of Child and Family Health. (2007). The importance of play in promoting healthy child development and maintaining strong parent-child bonds. Pediatrics, 119(1), 182-191. https://doi.org/10.1542/peds.2006-2697

Gobierno de España (2020). Real decreto 463/2020, de 14 de marzo, por el que se declara el estado de alarma para la gestión de la situación de crisis sanitaria ocasionada por el COVID-19. Madrid: BOE. https://doi.org/10.2307/j.ctt22nmcxk.10

Greve, W., Thomsen, T., \& Dehio, C. (2014). Does playing pay? the fitness-effect of free play during childhood. Evolutionary Psychology: An International Journal of Evolutionary Approaches to Psychology and Behavior, 12(2), 434-447. https://doi.org/10.1177/147470491401200210

Guhn, M., Emerson, S. D., \& Gouzouasis, P. (2020). A population-level analysis of associations between school music participation and academic achievement. Journal of Educational Psychology, 112(2), 308-328. https://doi.org/10.1037/edu0000376

Guthold, R., Stevens, G. A., Riley, L. M., \& Bull, F. C. (2018). Worldwide trends in insufficient physical activity from 2001 to 2016: A pooled analysis of 358 population-based surveys with 1.9 million participants. The Lancet Global Health, 6(10), e1077-e1086. https://doi.org/10.1016/s2214-109x(18)30357-7

Herazo-Beltran, Y., Campo-Ternera, L., Garcia-Puello, F., Mendez, O., Suarez-Villa, M., VasquezDe la Hoz, F., \& Nunez-Bravo, N. (2019). Relationship between physical activity and emotional intelligence and bullying among school children. Revista de Psicologia del Deporte, 28(1), $97-$ 103. https://doi.org/10.14482/sun.33.3.10931 
Hernández Pérez, F. (2019). Los riesgos de las tecnologías de la información y la comunicación. Revista CONAMED, v. 24, n. 4, pp. 184-189. https://www.medigraphic.com/pdfs/conamed/con2019/con194d.pdf

Hernández-Prados, M. Á., López-Vicent, P., \& Sánchez-Esteban, S. (2014). La comunicación en la familia a través de las TIC: Percepción de los adolescentes. Pulso: revista de educación, 37, 35-58. Recuperado de https://dialnet.unirioja.es/servlet/articulo?codigo=4954346

Instituto Nacional de Estadística (2019). Encuesta sobre Equipamiento y Uso de Tecnologías de Información y Comunicación en los Hogares. Madrid: INE.

Jover-Olmeda, G., Camas-Garrido, L., Martín-Ordanza Santos, P., \& Sánchez-Serrano, S. (2018). La contribución del juego infantil al desarrollo de habilidades para el cambio social activo. Madrid: Universidad Complutense de Madrid. https://doi.org/10.21840/siic/144243

Justicia-Justicia, F., \& Villadangos-González, S. M. (2010). Menores y nuevas tecnologías: Conductas indicadoras de posible problema de adición. Psicothema, 22(2), 180-188. http://www.psicothema.com/psicothema.asp?id=3713

Lázaro-Rodríguez, P. y Herrera-Viedma, E. (2020). Noticias sobre Covid-19 y 2019-nCoV en medios de comunicación de España: el papel de los medios digitales en tiempos de confinamiento. El profesional de la información, 29(3), 290-302. https://doi.org/10.3145/epi.2020.may.02

Lloret-Irles, D., \& Morell-Gomis, R. (2016). Impulsiveness and video game addiction. Health and Addictions: Salud y Drogas, 16(1), 33-40. https://doi.org/10.21134/haaj.v16i1.255

Lloret-Irles, D., Morell-Gomis, R., Marzo-Campos, J. C., \& Tirado-González, S. (2018). Validación española de la escala de adicción a videojuegos para adolescentes (GASA). Atención Primaria: Publicación Oficial de la Sociedad Española de Familia y Comunitaria, 50(6), 350-358. https://doi.org/10.1016/j.aprim.2017.03.015

Lozano-Sánchez, A., Zurita-Ortega, F., Ubago-Jiménez, J. L., Puertas-Molero, P., Ramírez-Granizo, I., \& Núñez-Quiroga, J. I. (2019). Videojuegos, práctica de actividad física, obesidad y hábitos sedentarios en escolares de entre 10 y 12 años de la provincia de granada. Retos: Nuevas Tendencias en Educación Física, Deporte y Recreación, 35, 42-46. https://doi.org/10.47197/retos.v0i35.61865

Lucas-Marín, A. (2006). Las tecnologías de la información y la comunicación: Cambios en el modelo de comunicación. Hologramática, v. 4, n. 1, pp. 15-33. https://doi.org/10.26820/recimundo/3.(3.esp).noviembre.2019.706-724

Maher, C., Virgara, R., Okely, T., Stanley, R., Watson, M., \& Lewis, L. (2019). Physical activity and screen time in out of school hours care: An observational study. BMC Pediatrics, 19(1), 283. https://doi.org/10.1186/s12887-019-1653-X

Marqués, Pere, (2012). Impacto de las TIC en la educación: funciones y limitaciones. 3C TIC. Revista de Investigación, 3, 1-15. https://www.3ciencias.com/articulos/articulo/impacto-de-las-ticen-la-educacion-funciones-y-limitaciones/

Ministerio de Educación y Formación Profesional. (2019). Sistema estatal de indicadores de la educación. Madrid: Instituto Nacional de Evaluación Educativa del Gobierno de España. http://www.educacionyfp.gob.es/dam/jcr:627dc544-8413-4df1-ae46-558237bf6829/seie-2019.pdf 
RLCS, Revista Latina de Comunicación Social, 78, 183-204

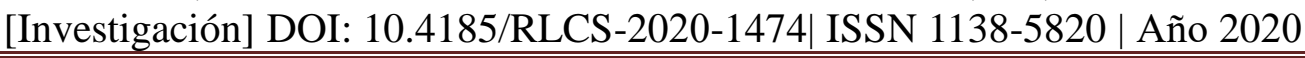

Nakamura, R., Yamashita, J., Akabayashi, H., Tamura, T., \& Zhou, Y. (2020). A comparative analysis of children's time use and educational achievement: Assessing evidence from China, Japan and the United States. Chinese Journal of Sociology, 6(2), 257-285, https://doi.org/10.1177/2057150x20911871

Organización Mundial de la Salud (2010). Estrategia mundial sobre régimen alimentario, actividad física y salud. Recomendaciones mundiales sobre actividad física para la salud. Ginebra: OMS. https://www.who.int/dietphysicalactivity/publications/9789241599979/es/

Organización Mundial de la Salud (2019). Guidelines on physical activity, sedentary behaviour and sleep for children under 5 years of age. OMS. https://doi.org/10.26719/2016.22.5.350

Organización Mundial de la Salud (2020). COVID-19: Cronología de la actuación de la WHO. https://www.who.int/es/news-room/detail/27-04-2020-who-timeline---covid-19

Ortiz Sobrino, M. Á., Marta Lazo, C. y Gabelas Barroso, J. A. (2019). Los niños y adolescentes ante las pantallas: el cambio de paradigma en el modelo de relaciones y mediación. Historia y Comunicación Social, 24(1), 353-365. https://doi.org/10.5209/hics.64499

Paterna-Bleda, C., \& Martínez-Martínez, M. C. (2009). Influencia de las variables de género en la distribución de las tareas domésticas y de cuidado. Interamerican Journal of Psychology, 43(2), 241-249. https://www.redalyc.org/pdf/284/28412891005.pdf

Perone, S., Anderson, A. J., \& Youatt, E. A. (2020). Don't forget your lunch: Age and individual differences in how children perform everyday tasks. Cognitive Development, 54. https://doi.org/10.1016/j.cogdev.2020.100879

Piercy, K. L., Troiano, R. P., Ballard, R. M., Carlson, S. A., Fulton, J. E., Galuska, D. A., ... Olson, R. D. (2018). The physical activity guidelines for americans. JAMA, 320(19), 2020-2028. https://doi.org/10.1001/jama.2018.14854

Piya-Amornphan, N., Santiworakul, A., Cetthakrikul, S., \& Srirug, P. (2020). Physical activity and creativity of children and youths. BMC Pediatrics, 20(118), 1-7. https://doi.org/10.1186/s12887$\underline{020-2017-2}$

Remorini, C., Teves, L. S., Palermo, M. L., Jacob, A., \& Desperés, P. (2019). Acerca de la participación de niños y niñas en actividades de subsistencia: Estudio etnográfico en unidades domésticas rurales de salta (argentina). Runa: Archivo para las Ciencias del Hombre, 40(2), 293312. https://doi.org/10.34096/runa.v40i2.5503

Rodríguez-Hernández, J. A., \& Santana-Bonilla, P. J. (2006). Maestras y maestros: Un análisis de la distribución de tareas docentes y domésticas. Revista de Educación, 340, 873-922. http://hdl.handle.net/11162/69056

Salinas, D. (2014). ¿Perpetúan los deberes las desigualdades en educación? PISA in Focus, 12, 1-4. https://www.educacionyfp.gob.es/inee/dam/jcr:8fd5734c-1633-4796-8796-e2e686e2df20/pisa-infocus-n46-esp.pdf

Sánchez-López, I., Pérez-Rodríguez, M. A., \& Fandos-Igado, M. (2019). Com-educational platforms: Creativity and community for learning. NAER: Journal of New Approaches in Educational Research, 8(2), 214-226. https://doi.org/10.7821/naer.2019.7.437

Sañudo, L. E. (2006). La ética en la investigación educativa. Hallazgos, 3(6). https://doi.org/10.15332/s1794-3841.2006.0006.05 
RLCS, Revista Latina de Comunicación Social, 78, 183-204

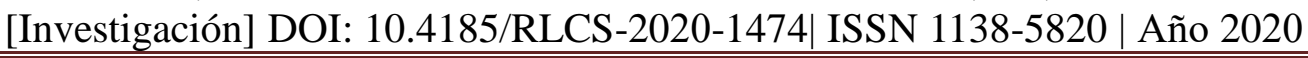

Scarafoni, M. E. (2016). División sexual del trabajo doméstico y el uso del tiempo en las tareas de cuidado. Derecho y Ciencias Sociales, 14, 127-136. https://doi.org/10.3989/ris.2009.08.26

Solito, L., \& Sorrentino, C. (2018). Political Communication and Challenges in the Digital Age. Revista ICONO14 Revista Científica de Comunicación y Tecnologías Emergentes, 16(1), 22-41. https://doi.org/10.7195/ri14.v16i1.1161

Tejeiro, R. (2001). La adicción a los videojuegos. una revisión. Adicciones: Revista De Socidrogalcohol, 13(4), 407-413. https://doi.org/10.20882/adicciones.555

Torrecillas-Lacave, T., Vázquez-Barrio, T., Suárez-Álvarez, Rebeca y Fernández-Martínez, L. M. (2020). El papel de los padres en el comportamiento online de menores hiperconectados. Revista Latina de Comunicación Social, 75, 121-148. https://doi.org/10.4185/rlcs-2020-1419

Torres-Moreno, P., Solera-Albero, J., Sahuquillo-Martínez, A., \& Tárraga-López, P. J. (2020). Influencia de una intervención comunitaria en obesidad y estilos de vida de escolares en el ámbito rural. JONNPR, 5(1), 1-136. http://dx.doi.org/10.19230/jonnpr.3205

Valle, A., Piñeiro, I., Rodríguez Martínez, S., Regueiro, B., Freire, C., \& Rosário, P. (2019). Time spent and time management in homework in elementary school students: A person-centered approach. Psicothema, 31(4), 422-428. https://doi.org/10.7334/psicothema2019.191

Yubero-Jiménez, S., \& Larrañaga-Rubio, E. (2010). El valor de la lectura en relación con el comportamiento lector. Un estudio sobre hábitos lectores y estilo de vida en niños. Revista OCNOS: Revista De Estudios Sobre Lectura, 6, 7-20. https://doi.org/10.18239/ocnos_2010.06.01

Zueck, M. d. C., Ramírez García, A. A., Rodríguez Villalobos, J. M., \& Irigoyen Gutiérrez, H. E. (2020). Satisfacción en las clases de educación física y la intencionalidad de ser activo en niños del nivel de primaria. Retos: Nuevas Tendencias en Educación Física, Deporte y Recreación, v. 37, pp. 33-40. https://doi.org/10.24054/16927427.v1.n1.2017.3355

\section{AUTORES:}

\section{Víctor Arufe Giráldez}

Víctor Arufe es profesor en la Facultad de Educación de la Universidad de A Coruña. Editor Jefe de la revista científica Sportis. Director de la Unidad de Investigación UNIDEF. Miembro del Comité de Expertos de Evaluación de Proyecto de Investigación de la Agencia Estatal de Investigación del Ministerio de Economía, Industria y Competitividad del Gobierno de España. Autor de más de 30 artículos científicos y numerosos libros publicados por diversas editoriales. Autor del Blog Educación, Innovación y Emprendimiento con más de 2000 visitas diarias. Ha participado en numerosos proyectos de investigación y ha participado como ponente invitado en más de 50 congresos nacionales e internacionales. Reconocimiento a la excelencia docente e investigadora de la ACSUG.

v.arufe@udc.es

Índice H: 13

Orcid ID: https://orcid.org/0000-0002-6440-048X

Google Scholar: https://scholar.google.es/citations?user=d42j4eQAAAAJ\&hl=es

ResearchGate: https://www.researchgate.net/profile/Victor_Arufe-Giraldez

Scopus ID: https://www.scopus.com/authid/detail.uri?authorId=56464204200

ResearcherID: https://publons.com/researcher/2490594/victor-victor-arufe-arufe-giraldez/ 


\section{Javier Cachón Zagalaz}

El Dr. Javier Cachón es profesor e investigador en la Facultad de Humanidades y Ciencias de la Educación de la UJA. Coordina la especialidad de EF en el Máster de Profesorado, en el que imparte clases, así como en el Grado de Educación Primaria. Forma parte del Programa de Doctorado en Innovación Didáctica y Formación del Profesorado, habiendo dirigido 9 tesis doctorales. Es autor de libros y capítulos relacionados en la base SPI de Humanidades y CCSS, así como de artículos en revistas JCR y Scopus. Ha participado como conferenciante y ponente invitado en congresos nacionales e internacionales. Coordina distintos programas Erasmus y forma parte de proyectos de investigación e innovación educativa coordinando alguno de ellos.

jcachon@ujaen.es

Índice H: 18

Orcid ID: https://orcid.org/0000-0001-5085-0423

Google Scholar: https://scholar.google.es/citations?user=WJb8GigAAAAJ\&hl=es

ResearchGate: https://www.researchgate.net/profile/Javier_Cachon-Zagalaz

Scopus ID: https://www.scopus.com/authid/detail.uri?authorId=55634498700

Academia.edu: https://independent.academia.edu/JavierCach\%C3\%B3nZagalaz

ResearcherID: https://publons.com/researcher/2090383/javier-jcz-cachon-zagalaz/

\section{María Luisa Zagalaz Sánchez}

María Luisa es catedrática en el área de la Didáctica de la Expresión Corporal. Presenta una dilatada trayectoria académica y científica con una importante producción. Ponente invitada en decenas de congresos nacionales e internacionales y autora de cerca de un centenar de artículos científicos con más de 3000 citas registradas en Google Scholar.

lzagalaz@ujaen.es

Índice H: 27

Orcid ID: https://orcid.org/0000-0001-6044-8569

Google Scholar: https://scholar.google.es/citations?user=uPfthgN73nUC\&hl=es

Scopus ID: https://www.scopus.com/authid/detail.uri?authorId=35276605200

\section{Alberto Sanmiguel-Rodríguez}

Alberto Sanmiguel-Rodríguez es profesor e investigador de la Universidad Antonio de Nebrija y en la Universidad Camilo. Personal laboral fijo del Estado como profesor titular. Ha participado en varias jornadas y congresos nacionales e internacionales, se destacan entre otros el $9^{\circ}$ Congreso nacional de ciencias de la actividad física y el deporte, el 15th IARTEM International celebrado en la UCL University College de Odense (Dinamarca). Ha participado como comité organizador y científico en el II y III Congreso Mundial de Educación EDUCA 2019 y 2020 respectivamente y en el 15 Congreso internacional de Ciencias del Deporte y la Salud 2019. Autor de numerosos artículos científicos.

asrgz2014@gmail.com

Orcid ID: https://orcid.org/0000-0001-5119-5141

Google Scholar: https://scholar.google.es/citations?user=tapqbBIAAAAJ\&hl=es\&oi=ao

ResearchGate: https://www.researchgate.net/profile/Alberto Sanmiguel-Rodriguez

Scopus ID: https://www.scopus.com/authid/detail.uri?authorId=57208429566

ResearcherID: https://publons.com/researcher/1858898/alberto-sanmiguel-rodriguez

Dialnet: https://dialnet.unirioja.es/servlet/autor?codigo=4355749 


\section{Gabriel González Valero}

Gabriel González Valero profesor de la Facultad de Humanidades y Ciencias de la Educación (Universidad de Jaén). Pertenece al grupo de investigación HUM-238 de la Universidad de Granada. Autor de numerosos artículos científicos del ámbito de la educación y las TIC.

Correo: ggvalero@ujaen.es

Índice H: 9

Orcid ID: https://orcid.org/0000-0001-7472-5694

Google Scholar: https://scholar.google.es/citations?hl=es\&user=C17xfdUAAAAJ

ResearchGate: https://www.researchgate.net/profile/Gabriel_Gonzalez_Valero

Scopus ID: 57204592697

Academia.edu: https://ugr.academia.edu/GabrielGonz\%C3\%A1lezValero

ResearcherID: G-9817-2018 